\title{
A continuum failure criterion applicable to wood
}

\begin{abstract}
The failure criterion is an essential part of all strength calculations of design. It was shown in the past that the tensor-polynomial equation could be regarded as a polynomial expansion of the real failure surface. Now it is shown that the third-degree polynomial is identical to the real failure criterion. It is also shown that the second-degree part of the polynomial is identical to the orthotropic extension of the von Mises criterion for initial yield. The thirddegree polynomial hardening terms of the criterion are also shown to incorporate the earlier theoretical explained mixed-mode I-II fracture equation, showing hardening to be based on hindered microcrack extension. For uniaxial loading, the failure criterion can be resolved in factors, leading to the derivation of extended Hankinson equations. This allows the relations between the constants of the total failure criterion to be elucidated, which is necessary for data fitting of this criterion and providing a simple method to determine the constants by the simple uniaxial, obliquegrain compression and tension tests. Based on this, the numerical failure criterion is given with the simple lower bound criterion for practice and for the codes.
\end{abstract}

Key words Timber · Failure criterion · Tensor-polynomial · Extended orthogonal von Mises criterion · Extended Hankinson equations

\section{Introduction}

Design and control calculations are normally based on limit analysis by finding an equilibrium system that satisfies the

T.A.C.M. van der Put

Division of Timber Structures and Wood Technology, Faculty of

Civil Engineering and Geosciences, Delft University of Technology,

PO Box 5048, Delft 2600 GA, The Netherlands

Tel. +31-15-2851980; Fax +31-15-2783173

e-mail: vanderp@xs4all.nl

Parts of this article in preliminary form were presented at the 15 th Conseil Industrielle des Bois-International Union of Forestry Research Organizations Meeting, Boras, Sweden, May 1982, and at the 3rd COST Meeting, Limerick, Ireland, April 1993 boundary conditions and nowhere surmounts the failure criterion. The derivation of the failure criterion for wood is given here, which is shown by van der $\mathrm{Put}^{1}$ to be identical to a polynomial expansion of the real failure criterion. The quadratic polynomial part of this polynomial is shown in the following section to represent the critical distortional energy criterion of initial yield and thus is not just an expansion, but is identical to the real yield criterion. It also is shown that the third-degree terms of the polynomial follow the theoretical explained mixed-mode I-II equation and represent special hardening effects by microcrack arrest. The fourth-degree and higher-degree polynomial terms have no meaning and are zero. As empirical confirmation, the clear wood results given by van der $\mathrm{Put}^{1}$ are discussed together with the biaxial data for timber from Hemmer. ${ }^{2}$

\section{Theory}

It was shown for the first time for wood by van der Put ${ }^{1}$ that the second-degree tensor-polynomial describes initial "flow," which is shown here to represent the extension of the critical distortional energy criterion to orthotropic materials providing the necessary basis for exact solutions according to limit analysis (see Discussion). Because an isotropic matrix of a material may sustain large hydrostatic pressures without yielding, yield depends on a critical value of the distortional energy. This energy $W_{\mathrm{d}}$ is found by subtracting the energy of the volume change from the total strain energy. Thus, for the isotropic matrix material, this is

$$
\begin{aligned}
W_{d}= & \left(\frac{1}{2 E} \cdot\left(\sigma_{x}^{2}+\sigma_{y}^{2}+\sigma_{z}^{2}\right)-\frac{v}{E} \cdot\left(\sigma_{x} \sigma_{y}+\sigma_{y} \sigma_{z}+\sigma_{z} \sigma_{x}\right)+\right. \\
& \left.\frac{1+v}{E} \cdot\left(\tau_{x y}^{2}+\tau_{y z}^{2}+\tau_{x z}^{2}\right)\right)-\left(\frac{1-2 v}{6 E} \cdot\left(\sigma_{x}+\sigma_{y}+\sigma_{z}\right)^{2}\right) \\
= & \frac{1+v}{6 E} \cdot\left(\left(\sigma_{x}-\sigma_{y}\right)^{2}+\left(\sigma_{y}-\sigma_{z}\right)^{2}+\left(\sigma_{z}-\sigma_{x}\right)^{2}\right)+ \\
& \frac{1}{2 G} \cdot\left(\tau_{x y}^{2}+\tau_{x z}^{2}+\tau_{y z}^{2}\right)
\end{aligned}
$$


where $\sigma_{i}$ are the normal matrix stresses; $\tau_{i}$ the shear stresses; $E$ the modulus of elasticity; $G$ the shear modulus; and $v$ Poison's ratio of the matrix material following $2 G=E /$ $(1+v)$.

Wood has to be regarded as a reinforced material and initial failure is due to failure of the isotropic matrix. This was shown by van der Put, ${ }^{3}$ leading to a new fracture mechanics theory and a new transformation of the Airy stress function. This makes exact solutions possible as applied for the derivations of the $\mathrm{Wu}^{4}$ mixed-mode I-II fracture criterion and the derivations of the right fracture energies and the relation between mode I and II stress intensities and energy release rates. According to van der Put, ${ }^{3}$ the matrix stresses can be expressed in orthotropic stresses as follows.

The stress in wood $\sigma_{x, o r}$ is $n_{1}$ times the stress in the matrix $\sigma_{x}$ due to the reinforcement in $x$-direction: $\sigma_{x, o r}=\left(E_{x} / E\right) \cdot \sigma_{x}$ $=n_{1} \cdot \sigma_{x}$, while the reinforcement in $y$-direction is regarded to belong to the matrix, thus $\sigma_{y, o r}=\sigma_{y}$ and $E_{y}=E$ of the matrix. For the shear stress, the multiplying factor is $n_{6}=$ $\left(2+v_{x y}+v_{y x}\right) \cdot G_{x y} / E$. Thus, $E_{x}, E_{y}, G_{x y}, v_{x y}$, and $v_{y x}$ are the orthotropic values of wood due to the reinforcements.

Equation 1 applies for a material with equal tension and compression strength. For unequal axial strengths, the failure condition, in $x$-direction is: $\left(\sigma_{x}-X\right) \cdot\left(\sigma_{x}+X^{\prime}\right)=0$, where $X$ is the tensile strength and $-X^{\prime}$ the compression strength, as given in Fig. 1b. This condition can be written as

$\left(\sigma_{x}-\frac{X-X^{\prime}}{2}\right)^{2}=\left(\frac{X+X^{\prime}}{2}\right)^{2}$ or: $\sigma_{x}-p_{x}= \pm \bar{X}$

(see Fig. 1) and the behavior is identical to that of a material with equal tension and compression strengths of $\bar{X}$ being prestressed by stress $p_{x}$.

This result follows from the applied linear transformation. Because Eq. 1 describes a physical property, it should be independent of the chosen vector space and according to the additivity rule of linear mapping (linear transformation) is

$\mathrm{f}(x+y)=\mathrm{f}(x)+\mathrm{f}(y)$,

or in this case:

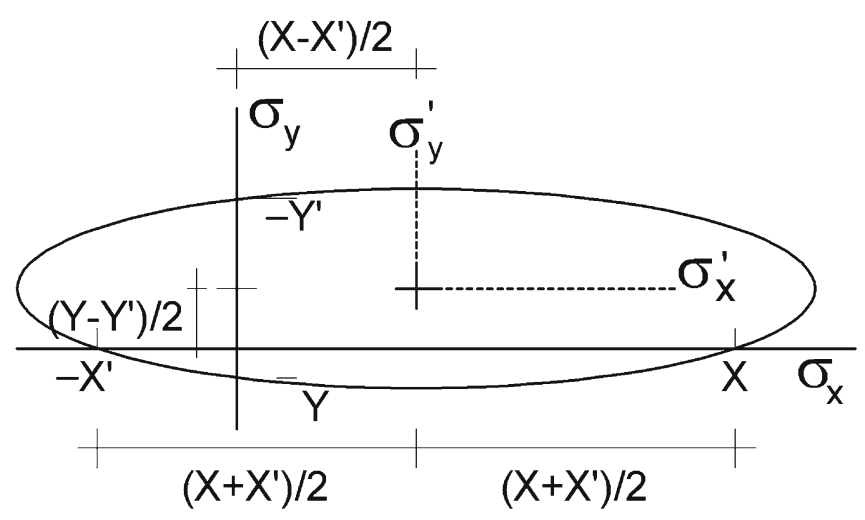

Fig. 1. von Mises criterion for wood $\mathrm{f}(\sigma-p)=\mathrm{f}(\sigma)+\mathrm{f}(-p)$ giving

$\mathrm{f}(\sigma)=\mathrm{f}(\sigma-p)-\mathrm{f}(-p)$

Substitution of $\sigma_{x}-p_{x}, \sigma_{y}-p_{y}$, and $\sigma_{z}-p_{z}$ for $\sigma_{x}, \sigma_{y}$, and $\sigma_{z}$, respectively in Eq. 1 gives

$$
\begin{aligned}
& \left(\frac{\sigma_{x, o r}}{n_{1}}-\frac{p_{x, o r}}{n_{1}}-\sigma_{y, o r}+p_{y}\right)^{2}+\left(\sigma_{y, o r}-p_{y}-\sigma_{z, o r}+p_{z}\right)^{2}+ \\
& \left(\sigma_{z, o r}-p_{y}-\frac{\sigma_{x, o r}}{n_{1}}+\frac{p_{x, o r}}{n_{1}}\right)^{2}+ \\
& 6 \Sigma \tau_{i j}^{2} / n_{i}=2 C\left(=6 E W_{d} /(1+v)\right)
\end{aligned}
$$

and after subtraction of $\mathrm{f}(-p)$ this is

$$
\begin{aligned}
& \frac{\sigma_{x, o r}^{2}}{n_{1}^{2}}+\sigma_{y, o r}^{2}+\sigma_{z, o r}^{2}-\frac{\sigma_{x, o r} \sigma_{y, o r}}{n_{1}}-\sigma_{z, o r} \sigma_{y, o r}-\frac{\sigma_{x, o r} \sigma_{z, o r}}{n_{1}}+ \\
& \left(p_{y}+p_{z}-\frac{2 p_{x, o r}}{n_{1}}\right) \frac{\sigma_{x, o r}}{n_{1}}+\left(p_{y}-2 p_{z}+\frac{p_{x, o r}}{n_{1}}\right) \sigma_{z, o r}+ \\
& \quad\left(p_{z}-2 p_{y}+\frac{p_{x, o r}}{n_{1}}\right) \sigma_{y}+3 \Sigma \tau_{i j}^{2} / n_{i}=-C_{p}+\mathrm{f}(-p)+C \\
& \quad=C=3 E W_{d} /(1+v)
\end{aligned}
$$

with

$\mathrm{f}(-p)=p_{x, o r}^{2} / n_{1}^{2}+p^{2}+p_{z}^{2}-p p_{x, o r} / n_{1}-p p_{z}-p_{z} p_{x, o r} / n_{1}=C_{p}$

following from inserting $\sigma_{x}=p_{x}, \sigma_{y}=p_{y}=p$, and $\sigma_{z}=p_{z}$ in Eq. 1.

Of interest for failure by flat crack propagation is the plane stress equation with $\sigma_{z}=p_{z}=0 ; \tau_{x z}=\tau_{y z}=0$; and $p_{y}=p_{y, o r}=p$, giving for Eq. 4 :

$$
\begin{aligned}
& \frac{\sigma_{x, o r}^{2}}{C^{\prime} \cdot n_{1}^{2}}-\frac{\sigma_{x, o r} \cdot \sigma_{y, o r}}{C^{\prime} \cdot n_{1}}+\frac{\sigma_{y, o r}^{2}}{C^{\prime}}+\frac{\sigma_{x, o r}}{C^{\prime} \cdot n_{1}}\left(p-2 \frac{p_{x, o r}}{n_{1}}\right)- \\
& \frac{\sigma_{y}}{C^{\prime}}\left(2 p-\frac{p_{x, o r}}{n_{1}}\right)+3 \frac{\tau_{o r}^{2}}{C^{\prime} \cdot n_{6}^{2}}=1
\end{aligned}
$$

For $\sigma_{y, o r}=\tau_{o r}=0$, Eq. 5 becomes

$$
\frac{\sigma_{x, o r}^{2}}{C^{\prime} \cdot n_{1}^{2}}+\frac{\sigma_{x, o r}}{C^{\prime} \cdot n_{1}}\left(p-2 \frac{p_{x, o r}}{n_{1}}\right)=1
$$

This is identical to

$$
\left(\sigma_{x, o r}-X\right)\left(\sigma_{x, o r}+X^{\prime}\right)=0 \text {, }
$$

or to

$$
\sigma_{x, o r}^{2}+\left(X^{\prime}-X\right) \sigma_{x, o r}-X X^{\prime}=0,
$$

showing that

$$
\left(p n_{1}-2 p_{x, o r}\right)=X^{\prime}-X \text {, and } C^{\prime} \cdot n_{1}^{2}=X X^{\prime}
$$

The same applies in the perpendicular $y$-direction for the uniaxial tension and compression strengths $Y$ and $Y^{\prime}$ giving $C^{\prime}=Y Y^{\prime}$ and $\left(p_{x, o r} / n_{1}-2 p\right)=Y^{\prime}-Y$. This last result is to be expected because according to the molecular theory, the strength is proportional to the $E$ modulus and thus is $Y Y^{\prime}=X X^{\prime} / n_{1}^{2}$ and $X^{\prime}-X=n_{1}\left(Y-Y^{\prime}\right)$. Then it also is $p=p_{x, o r} / n_{1}=Y-Y^{\prime}=\left(\mathrm{X}-X^{\prime}\right) / n_{1}$.

Equation 5 becomes

$$
\frac{\sigma_{x, o r}^{2}}{C^{\prime} \cdot n_{1}^{2}}-\frac{\sigma_{x, o r} \cdot \sigma_{y, o r}}{C^{\prime} \cdot n_{1}}+\frac{\sigma_{y, o r}^{2}}{C^{\prime}}-p \frac{\sigma_{x, o r}}{C^{\prime} \cdot n_{1}}-p \frac{\sigma_{y, o r}}{C^{\prime}}+3 \frac{\tau_{o r}^{2}}{C^{\prime} \cdot n_{6}^{2}}=1
$$


or

$$
\begin{gathered}
\frac{\sigma_{x, o r}^{2}}{X X^{\prime}}+\frac{\sigma_{x, o r}}{X}-\frac{\sigma_{x, o r}}{X^{\prime}}-2 F_{12} \sigma_{x, o r} \sigma_{y, o r}+ \\
\frac{\sigma_{y, o r}^{2}}{Y Y^{\prime}}+\frac{\sigma_{y, o r}}{Y}-\frac{\sigma_{y, o r}}{Y^{\prime}}+\frac{\tau_{o r}^{2}}{S^{2}}=1
\end{gathered}
$$

where $S$ is the shear strength and

$2 F_{12}=1 / C^{\prime} n_{1}=1 / \sqrt{X X^{\prime} Y Y^{\prime}}$

This value of $F_{12}$ applies for initial stress redistribution and microcracking of the matrix and becomes lower by further straining reaching a near-zero value at final yield or failure initiation. This shows early dissipation of the elastic distortional energy, as also is indicated by the orthotropic finite element calculation of Gopu. ${ }^{5}$ This dissipation of distortional energy is according to the incompressibility condition and thus follows a minimum energy principle of yield. At the end of this stress redistribution, final yield occurs according to Eq. 7 with $F_{12}=0$, leading to a combined critical strain energy criterion of the reinforcements with a Tresca criterion of the matrix (see Discussion for the implications).

\section{Materials and methods}

The parameter estimation of the first general failure criterion of wood by van der $\mathrm{Put}^{1}$ was mainly based on uniaxial off-axis tension and compression tests on spruce like European softwoods and other species from data in the literature. ${ }^{6,7}$ Because this criterion also applies for uniaxial loading, the relations, according to Eq. 18 between the parameters, has to be satisfied and it will be shown that therefore the uniaxial tests are sufficient for the estimation of all parameters. For the empirical verification of this, an initial cooperative project was started for biaxial parameter estimation, and tests in the longitudinal-tangential plane of spruce like European white fir were done by Hemmer ${ }^{2}$ on torsion tube tests, subjected to tension and internal pressure. However, the verification of Eq. 18 is lacking in literature and is therefore given here, based on these data. The parameter estimation in all cases was based on fitting the Tsai-Wu equation, ${ }^{8}$ which can be regarded as the tensorpolynomial expansion of the real failure equation and has the form:

$$
\begin{aligned}
& F_{i} \sigma_{i}+F_{i j} \sigma_{i} \sigma_{j}+F_{i j k} \sigma_{i} \sigma_{j} \sigma_{k}+\ldots \ldots \ldots=1 \\
& \quad(i, j, k=1,2,3,4,5,6)
\end{aligned}
$$

where $\sigma$ (see Fig. 2) and $F$ are stress and strength tensors. In van der Put, ${ }^{1}$ it is shown that clear wood can be regarded as orthotropic in the main planes and the principal directions of the strengths are orthogonal (showing the common tensor transformations), and third-degree terms represent local hardening in stable tests leading for plane stress to

$$
\begin{gathered}
F_{1} \sigma_{1}+F_{2} \sigma_{2}+F_{11} \sigma_{1}^{2}+2 F_{12} \sigma_{1} \sigma_{2}+F_{22} \sigma_{2}^{2}+F_{66} \sigma_{6}^{2}+3 F_{112} \sigma_{1}^{2} \sigma_{2}+ \\
3 F_{221} \sigma_{2}^{2} \sigma_{1}+3 F_{166} \sigma_{1} \sigma_{6}^{2}+3 F_{266} \sigma_{2} \sigma_{6}^{2}+12 F_{1266} \sigma_{1} \sigma_{2} \sigma_{6}^{2}=1
\end{gathered}
$$

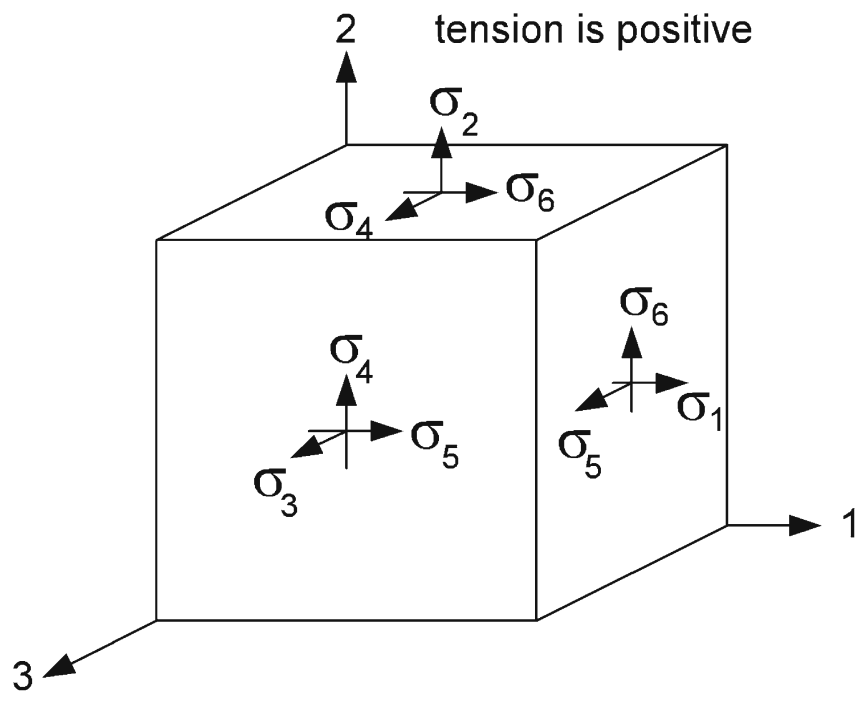

Fig. 2. Definition of positive stresses

For less stable tests, failure occurs at yield and $F_{12}$ is small and higher-degree hardening terms are negligible leading to Eq. 11, the critical distortional energy equation of initial yield derived before:

$$
F_{1} \sigma_{1}+F_{2} \sigma_{2}+F_{11} \sigma_{1}^{2}+2 F_{12} \sigma_{1} \sigma_{2}+F_{22} \sigma_{2}^{2}+F_{66} \sigma_{6}^{2}=1
$$

The relations between the parameters and the requirements for parameter estimation by uniaxial off-axis tests is as follows. By the uniaxial stress $\sigma_{t}$, the stresses in the main planes are

$\sigma_{1}=\sigma_{t} \cos ^{2} \theta \quad \sigma_{2}=\sigma_{t} \sin ^{2} \theta \quad \sigma_{6}=\sigma_{t} \cos \theta \sin \theta$

Substitution in Eq. 11 gives

$F_{1} \sigma_{t} \cos ^{2} \theta+F_{2} \sigma_{t} \sin ^{2} \theta+F_{11} \sigma_{t}^{2} \cos ^{4} \theta+$

$$
\left(2 F_{12}+F_{66}\right) \sigma_{t}^{2} \cos ^{2} \theta \sin ^{2} \theta+F_{22} \sigma_{t}^{2} \sin ^{4} \theta=1
$$

and expressed in the uniaxial tension and compression strengths $\left(X, X^{\prime}, Y, Y^{\prime}\right)$ and shear strength $S$, this becomes

$$
\begin{aligned}
& \sigma_{t} \cos ^{2} \theta\left(\frac{1}{X}-\frac{1}{X^{\prime}}\right)+\sigma_{t} \sin ^{2} \theta\left(\frac{1}{Y}-\frac{1}{Y^{\prime}}\right) \\
& +\frac{\sigma_{t}^{2} \cos ^{4} \theta}{X X^{\prime}}+2 F_{12} \sigma_{t}^{2} \sin ^{2} \theta \cdot \cos ^{2} \theta+\frac{\sigma_{t}^{2} \sin ^{4} \theta}{Y Y^{\prime}}+ \\
& \frac{\sigma_{t}^{2} \cos ^{2} \theta \sin ^{2} \theta}{S^{2}}=1
\end{aligned}
$$

The critical distortional energy equation of initial yield, Eq. 13 can be resolved into factors as follows

$\left(\frac{\sigma_{t} \cos ^{2} \theta}{X}+\frac{\sigma_{t} \sin ^{2} \theta}{Y}-1\right) \cdot\left(\frac{\sigma_{t} \cos ^{2} \theta}{X^{\prime}}+\frac{\sigma_{t} \sin ^{2} \theta}{Y^{\prime}}+1\right)=0$

giving the product of the Hankinson equations for tension and for compression, (where $X$ and $X^{\prime}$ are the strengths in the grain direction). This theoretical derivation of the empirical Hankinson equations applies according to van der Put $^{1}$ because

$2 F_{12}+1 / S^{2}=1 / X^{\prime} Y+1 / X Y^{\prime}$ 
The same can be done with Eq. 10 leading to

$$
\begin{aligned}
& F_{1} \sigma_{t} \cos ^{2} \theta+F_{2} \sigma_{t} \sin ^{2} \theta+F_{11} \sigma_{t}^{2} \cos ^{4} \theta+\left(2 F_{12}+F_{66}\right) \sigma_{t}^{2} \cos ^{2} \theta+ \\
& F_{22} \sigma_{t}^{2} \sin ^{4} \theta+3\left(F_{112}+F_{166}\right) \sigma_{t}^{3} \cos ^{4} \theta \cdot \sin ^{2} \theta+ \\
& \quad 3\left(\mathrm{~F}_{122}+\mathrm{F}_{266}\right) \sigma_{t}^{3} \sin ^{4} \theta \cdot \cos ^{2} \theta+12 \mathrm{~F}_{1266} \sigma_{t}^{4} \cos ^{4} \sin ^{4} \theta=1
\end{aligned}
$$

This fourth-degree equation in $\sigma_{t}$ can be written as the product of two quadratic equations

$$
\begin{aligned}
& \left(\frac{\sigma_{t} \cos ^{2} \theta}{X}+\frac{\sigma_{t} \sin ^{2} \theta}{Y}-1+\sigma_{t}^{2} \sin ^{2} \theta \cos ^{2} \theta \cdot C_{t}\right) . \\
& \left(\frac{\sigma_{t} \cos ^{2} \theta}{X^{\prime}}+\frac{\sigma_{t} \sin ^{2} \theta}{Y^{\prime}}+1+\sigma_{t}^{2} \sin ^{2} \theta \cdot \cos ^{2} \theta \cdot C_{d}\right)=0
\end{aligned}
$$

giving the Hankinson equations extended with terms containing hardening parameters $C_{t}$ and $C_{d}$. For a real failure surface the roots will be real and because one of the quadratic equations gives the roots for compression and the other for tension, which should be valid for zero values of $C_{t}$ and/or $C_{d}$ as well (reducing then to Eq. 14), the constants $C_{t}$ and $C_{d}$ are uniquely determined.

Performing the multiplication of Eq. 17, the parameters of Eq. 16 are known:

$$
\begin{aligned}
& 2 F_{12}=1 / X^{\prime} Y+1 / X Y^{\prime}-1 / S^{2}+C_{t}-C_{d}=C_{t}-C_{d} ; \\
& 3\left(F_{112}+F_{166}\right)=C_{t} / X^{\prime}+C_{d} / X ; \\
& 3\left(F_{122}+F_{266}\right)=C_{t} / Y^{\prime}+C_{d} / Y ; \text { and } 12 F_{1266}=C_{t} C_{d}
\end{aligned}
$$

Equation 16 is equal to the empirical criterion of van der Put $^{1}$ and Hemmer, ${ }^{2}$ which was based purely on data fitting to the tensor-polynomial, showing that $F_{1122}$ and other possible higher-degree terms are zero or negligible (e.g., $F_{1266}$ ). This is derived here theoretically, based on the theoretically explained hardening terms and Eq. 16 is the exact failure criterion of limit analysis. The values $C_{t}$ and $C_{d}$ (depending on the stability of the test) follow from fitting the extended Hankinson equations for uniaxial off-axis tension and compression.

Theoretically, $F_{166}=0$ because $\sigma_{1}$ is in the plane of the crack and is not influenced by the flat crack. For the same reason $F_{122}=0$. Given that $F_{122}=F_{166}=0$, the other constants follow from Eq. 18. This will be measurable on perfect straight clear specimens. For tension, $F_{266}=0$ according to Eq. 18. This is confirmed by the clear wood measurements with $n=2$ in Eq. 34 (see Discussion). Timber specimens with defects show deviations of the main planes from the in-plane stress direction and there are always stresses perpendicular to the flat crack plane such that the interaction terms $F_{122}$ and $F_{166}$ will not be zero. The high value of $F_{112}$ and the similar behavior of $F_{266}$ indicate that, due to the disturbances, random inclined initial cracks outside the main planes, are determining showing an extension of the in-plane $\mathrm{Wu}$ equation to three dimensions. As shown and discussed below, it is possible to determine $F_{266}$ and $F_{112}$ directly and $F_{166}$ and $F_{122}$ then follow from Eq. 18 .

As shown in van der Put, ${ }^{1} F_{12}$ is very small or zero for clear wood. The Hemmer ${ }^{2}$ data for biaxial strengths in longitudinal tension also show for timber that $F_{12}$ is zero at yield (see Fig. 3 showing zero slope of the ellipse) and thus is

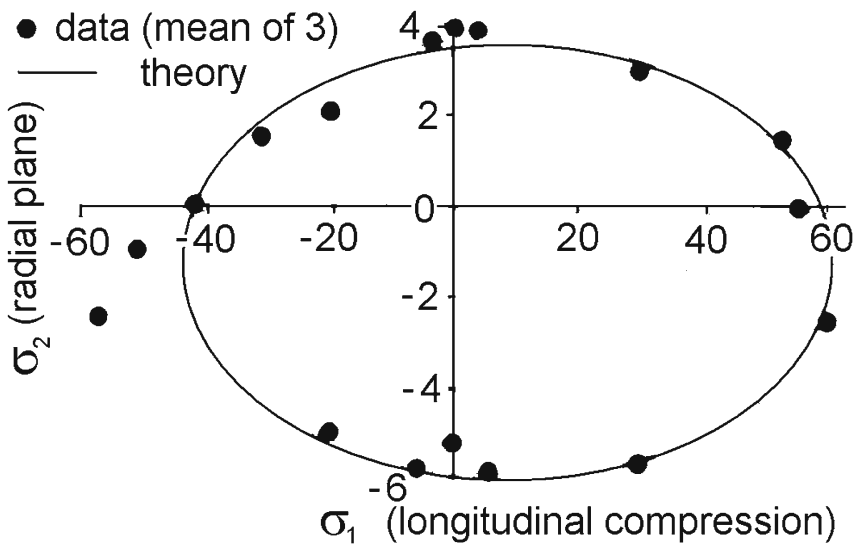

Fig. 3. Yield criterion for $F_{12}=0$ and $\sigma_{6}=0$

$1 / S^{2}=1 / X^{\prime} Y+1 / X Y^{\prime}$

Equation 18 now shows that, due to hardening, $F_{12}$ changes from zero at yield to

$2 F_{12}=C_{t}-C_{d}$

\section{Results}

Transverse strength

In van der $\mathrm{Put}^{1}$ it is shown that the quadratic polynomial like Eq. 11 precisely describes the peculiar flow behavior of the transverse compression-tension strength and (rolling) shear strength perpendicular to the grain $\tau_{\text {rol }}$ without the need for higher-order terms. After some strain hardening, the differences between radial-tangential compression strength and off-axes compression strength disappear and one directionally independent strength value remains. The behavior then becomes quasi isotropic and indicates the isotropic matrix to be determining. This also applies for the second hardening stage after the empty spaces in wood are pressed away. For tension perpendicular to the grain, only in a rather small region (around $90^{\circ}$, see Fig. 8 of van der $\mathrm{Put}^{1}$ in the radial direction) is the strength higher, making it negligible in practice such that a lower bound of strength will apply that is independent of the direction.

\section{Longitudinal strengths}

The longitudinal shear strength in the radial plane increases with compression perpendicular to this plane according to the coupling term $F_{266}$ (direction 2 is radial direction and direction 1 is grain direction):

$$
\begin{gathered}
F_{2} \sigma_{2}+F_{22} \sigma_{2}^{2}+F_{66} \sigma_{6}^{2}+3 F_{266} \sigma_{2} \sigma_{6}^{2}=1 \text { or } \\
\frac{\sigma_{6}}{S}=\sqrt{\frac{\left(1-\sigma_{2} / Y\right) \cdot\left(1+\sigma_{2} / Y^{\prime}\right)}{1+c \sigma_{2} / Y^{\prime}}}
\end{gathered}
$$

with $c=3 F_{266} Y^{\prime} S^{2} \approx 0.9$ (0.8 to 0.99 , see Fig. 4$)$. The value of $c$ depends on the stability of the test. When $c$ approaches 
$c \approx 1$ (measurements of project $\mathrm{A}$ in Fig. 4), Eq. 21 becomes

$\left(\frac{\sigma_{6}}{S}\right)^{2}+\frac{\sigma_{2}}{Y} \approx 1$

being the $\mathrm{Wu}$ equation of fracture mechanics theoretically explained by van der Put. ${ }^{3}$

Equation 21 is thus shown to be an exact equation. As derived in van der Put, ${ }^{3}$ Eq. 22 does not only apply for tension with shear but also for shear with compression $\sigma_{2}$ perpendicular to the flat crack plane. For a high stress $\sigma_{2}$, the crack is closed at $\sigma_{2}=\sigma_{c}$ and the crack tip notices only the influence of $\sigma_{2}=\sigma_{c}$ because for the higher part of $\sigma_{2}$, the load is directly transmitted through the closed crack and Eq. 22 becomes

$$
\frac{\sigma_{6}}{S}=\frac{-\mu\left(\sigma_{2}-\sigma_{c}\right)}{S}+\sqrt{1-\frac{\sigma_{c}}{Y}} \text { or } \quad \sigma_{6}=C+\mu\left|\sigma_{2}\right|
$$

where $\sigma_{2}$ and $\sigma_{c}$ are negative, giving the Coulomb equation with an increased shear capacity due to friction: $\mu\left|\sigma_{2}\right|$. However, inserting the measured values of Hemmer, ${ }^{2}$ it appears that the frictional contribution is very small. The microcrack closure stress $\sigma_{c}$ will be about equal to the tensile strength: $\sigma_{c} \approx-Y$. The shear strength will be maximal raised, at high compression of $\sigma_{c} \approx-0.9 Y^{\prime}$, by a factor

$$
\begin{aligned}
\left(1+\mu\left(0.9 Y^{\prime}-Y\right) / S \sqrt{2}\right) & =(1+0.3(0.9 \cdot 5.6-3.7) / 9.8 \cdot \sqrt{2}) \\
& =1.03
\end{aligned}
$$

Thus, the combined shear-compression strength is mainly determined by an equivalent hardening effect, caused by crack arrest in the critical direction by strong layers. At higher $\sigma_{2}$ stresses, compression plasticity perpendicular to the grain (project A of Keenan and Jaeger, ${ }^{7}$ see Fig. 4 ), or instability of the test (project B of Keenan and Jaeger $^{7}$ of oblique-grain compression tests) may become determining.

The parabolic strength behavior at biaxial compression in the main planes by $\sigma_{1}$ and $\sigma_{2}$, with $\sigma_{6}=0$, determined by $F_{112}$, given by the longitudinal compression data outside the drawn ellipse in Fig. 3, can also be explained by crack propagation due to initial off-axis microcracks, giving an

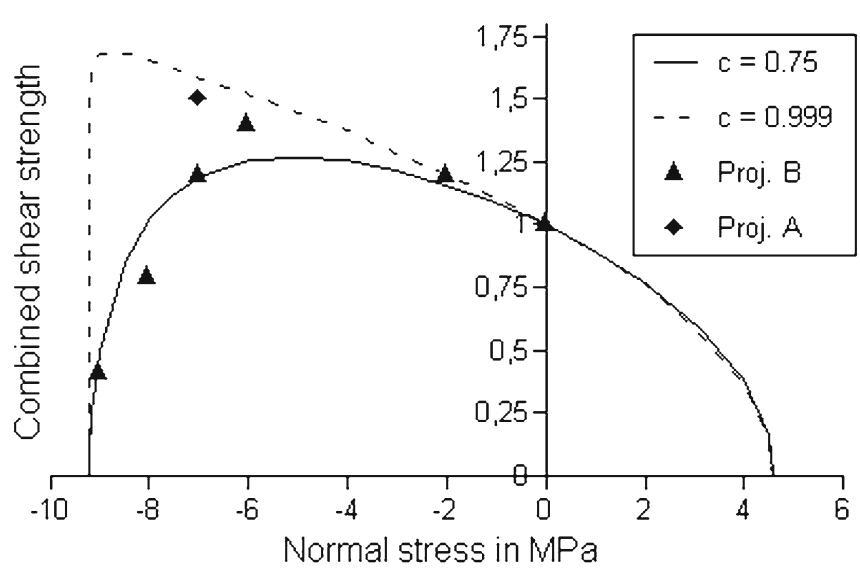

Fig. 4. Combined shear-tension and shear-compression strengths extension of the $\mathrm{Wu}$ equation to the biaxial loading case. $F_{112}$ has the most influence when $\sigma_{6}=0$ and when the theoretical value $F_{122}=0$ is reached; then the failure equation is

$\sigma_{1}\left(\frac{1}{X}-\frac{1}{X^{\prime}}\right)+\sigma_{2}\left(\frac{1}{Y}-\frac{1}{Y^{\prime}}\right)+\frac{\sigma_{1}^{2}}{X X^{\prime}}+\frac{\sigma_{2}^{2}}{Y Y^{\prime}}+3 F_{112} \sigma_{2} \sigma_{1}^{2}=1$

This can be written as

$$
\begin{gathered}
\sigma_{1}\left(X^{\prime}-X\right)+\sigma_{1}^{2}\left(1+3 F_{112} \sigma_{2} X X^{\prime}\right) \\
\quad=\left(1-\sigma_{2} / Y\right) \cdot\left(1+\sigma_{2} / Y^{\prime}\right) \cdot X X^{\prime}
\end{gathered}
$$

The critical value of $F_{112}$, to just have a closed failure criterion surface, will occur at high absolute values of $\sigma_{1}$ and $\sigma_{2}$, thus in the neighborhood of $\sigma_{1} \approx-X^{\prime}$. Inserting this value in the smallest term of Eq. 25 gives

$$
\begin{aligned}
& \sigma_{1}^{2}\left(1+3 F_{112} \sigma_{2} X X^{\prime}+\left(X^{\prime}-X\right) /\left(-X^{\prime}\right)\right) \\
& \quad=\left(1-\sigma_{2} / Y\right) \cdot\left(1+\sigma_{2} / Y^{\prime}\right) \cdot X X^{\prime}
\end{aligned}
$$

or

$$
\frac{\sigma_{1}}{X^{\prime}}=\sqrt{\frac{\left(1-\sigma_{2} / Y\right) \cdot\left(1+\sigma_{2} / Y^{\prime}\right)}{1+c \sigma_{2} / Y^{\prime}}} \quad \text { where } c=3 F_{112} Y^{\prime} X^{\prime 2}
$$

Thus, when $c=1$, the curve reduces to a parabola and the requirement to have a closed failure curve is $c<1$. Thus,

$3 F_{112}<1 / Y^{\prime} X^{\prime 2}$.

For longitudinal tension $\sigma_{1}$, the right side of Fig. 3 is an ellipse, which corresponds to a second-degree equation with zero $F_{112}$ and $F_{122}$ due to instability of the test.

It is not possible to have one precise overall fit for the failure criterion for the different failure types. This is because the values of the third-degree hardening terms depend on the loading state and separate fits are necessary for longitudinal tension with zero $F_{112}, F_{12}$, and $F_{122}$ and for longitudinal compression with hardening, whereby, $F_{112}$ dominates and $F_{12}$ is not zero. With the estimates of $F_{266}$ and $F_{112}$ close to their bounds for compression, and with zero normal coupling terms for tension, all constants of one general failure criterion, Eq. 16, are known according to Eq. 18 , depending on the fitted values of $C_{d}$ and $C_{t}$ from uniaxial off-axis tension and compression tests.

Estimation of polynomial constants by uniaxial tests

Based on data fitting of uniaxial tension and compression tests of Hemmer, ${ }^{2}$ the values of $C_{d}$ and $C_{t}$ are determined here. These are compared with the data and fit of the biaxial measurements of Hemmer. ${ }^{2}$

In Fig. 5 of Hemmer, ${ }^{2}$ the drawn line and dashed line in the figure give the prediction of the uniaxial values based on the biaxial measured constants according to the general Eq. 16. For comparison, the fits of the extended Hankinson equations are also given here. For tension, by scratching the nonzero compression term of the product, the extended Hankinson equation (Eq. 17) becomes

$$
\frac{\sigma_{t} \cos ^{2} \theta}{X}+\frac{\sigma_{t} \sin ^{2} \theta}{Y}+\sigma_{t}^{2} \sin ^{2} \theta \cos ^{2} \theta \cdot C_{t}=1
$$



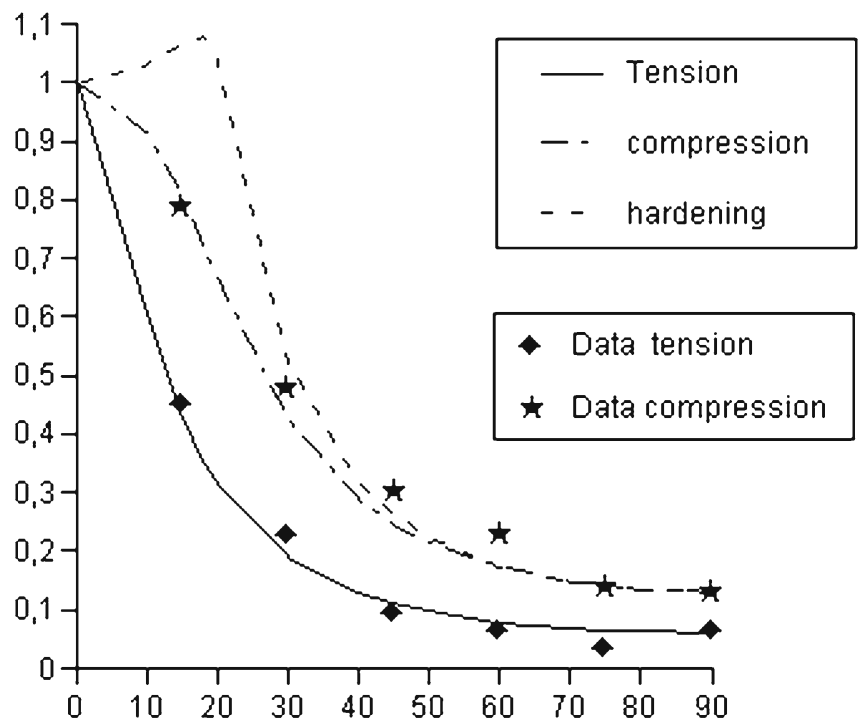

Fig. 5. Uniaxial tension strength and compression strength

This equation fits the line for tension in Fig. 5 when $C_{t} \approx 11.9 / X^{2}$. The Hankinson power equation (Eq. 34) fits in this case for $n \approx 1.8$ and Eqs. 16, 28, and 34 give the same result by only one independent constant $n$ or $C_{t}$.

For compression, the dashed-dot line according to Eq. 29 is drawn through the measured points at $90^{\circ}$ and $15^{\circ}$, giving the expected Hankinson power value of $n=2.4$ in Eq. 34 and $C_{d} \approx 4 /\left(X^{\prime}\right)^{2}$ in

$$
\frac{\sigma_{t} \cos ^{2} \theta}{X^{\prime}}+\frac{\sigma_{t} \sin ^{2} \theta}{Y^{\prime}}+\sigma_{t}^{2} \sin ^{2} \theta \cos ^{2} \theta \cdot C_{d}=1
$$

The dashed line in Fig. 5, based on the best fit of the biaxial measurements of Hemmer, ${ }^{2}$ is not a good fit for the oblique grain test. This deviation is due to early instability of the uniaxial off-grain test. This follows because, for example, the ratio of the compression strengths perpendicular to the grain and along the grain is 0.2 in the uniaxial tests and 0.1 in the biaxial tests, showing more hardening in the biaxial tests. Thus, strong hardening in the biaxial test will not occur under all circumstances and the hardening parameters should be omitted for a consistent safe lower bound criterion.

A fit of the longitudinal tensile data of $\mathrm{Hemmer}^{2}$ by the second-degree polynomial (ellipse) in the principal stresses $\sigma_{1}$ and $\sigma_{2}\left(\sigma_{6}=0\right)$ (see Fig. 3) shows $F_{12}=0$ and $F_{112}=F_{122}=0$ in the radial plane. Because the Hankinson power value for tension $n$ is different from $n=2$, there must be higher-degree terms for shear $\left(F_{166}, F_{266}\right)$. The strength values according to the data fit in $\mathrm{Hemmer}^{2}$ are (in $\mathrm{N} / \mathrm{mm}^{2}$ ):

$$
\begin{aligned}
& X=59.5 ; X^{\prime}=41.7 ; Y=3.5 ; Y^{\prime}=-5.9 ; S \approx 9.7 \text { and } \\
& C_{t}=11.9 / X^{2} ; C_{d}=4 / X^{\prime 2}
\end{aligned}
$$

$F_{1266}$ can be neglected. It only provides a slight local correction.
The data fit for longitudinal tension with $F_{12}=F_{112}=F_{122}$ $=0$, predicts a uniaxial compression strength of $X^{\prime}=$ 41.7 MPa, giving the failure at the same hardening state as in the oblique grain test (where strong biaxial compression hardening does not occur). The constants are

$$
\begin{aligned}
& C_{t}=11.9 / X^{2}=11.9 / 59.5^{2}=0.00336 ; \\
& C_{d}=4 / X^{\prime 2}=4 / 41.7^{2}=0.00230
\end{aligned}
$$

leading by Eq. 18 to Eq. 32 giving a better fit than the best fit of Hemmer, ${ }^{2}$ even in the compression range due to the application of Eq. 18 and hardening constants.

In the same way, the data fit for longitudinal compression, based on the strength values of the best fit of Hemmer, ${ }^{2}$ (for comparison of the results), with $c$ values of $c_{112}=0.8$ and $c_{266}=0.9$ (starting point) leads to Eq. 31 .

Because $C_{t}, C_{d}$ and $n$ values of the Hankinson equations are sufficiently close to the published extreme values of $n$ for wood with defects, the $c$ values used here also can be used in general for all softwoods. For compression, Eq. 16 becomes

$$
\begin{aligned}
& \frac{\sigma_{6}^{2}}{S^{2}} \cdot\left(1+0.9 \cdot \frac{\sigma_{2}}{Y^{\prime}}+0.2 \cdot \frac{\sigma_{1}}{X^{\prime}}\right)=\left(1-\frac{\sigma_{1}}{X}\right) \cdot\left(1+\frac{\sigma_{1}}{X^{\prime}}\right)+ \\
& \quad\left(1-\frac{\sigma_{2}}{Y}\right) \cdot\left(1+\frac{\sigma_{2}}{Y^{\prime}}\right)- \\
& \left(1+0.8 \cdot \frac{\sigma_{2} \sigma_{1}^{2}}{Y^{\prime} X^{\prime 2}}-0.77 \cdot \frac{\sigma_{1} \sigma_{2}^{2}}{X^{\prime} Y^{\prime 2}}-0.41 \cdot \frac{\sigma_{1} \sigma_{2}}{X^{\prime} Y^{\prime}}\right)
\end{aligned}
$$

Based on the fit for longitudinal tension $\left(\sigma_{1} \geq 0\right)$, Eq. 16 becomes

$$
\begin{aligned}
& \frac{\sigma_{6}^{2}}{S^{2}} \cdot\left(1+0.68 \cdot \frac{\sigma_{2}}{Y^{\prime}}+0.47 \cdot \frac{\sigma_{1}}{X^{\prime}}\right)=\left(1-\frac{\sigma_{1}}{X}\right) \cdot\left(1+\frac{\sigma_{1}}{X^{\prime}}\right)+ \\
& \left(1-\frac{\sigma_{2}}{Y}\right) \cdot\left(1+\frac{\sigma_{2}}{Y^{\prime}}\right)-1
\end{aligned}
$$

giving a better overall fit than Eq. 31. Compression hardening according to Eq. 31 occurs for low values of $\sigma_{6}$ only, and only in the torsion tube test in the radial plane. Thus, Eq. 32 more generally represents the failure criterion for tension and compression in praxis when $n \neq 2$ (in Eq. 34).

For tests and structures, showing early instability and no hardening at failure in most situations in practice (causing the Hankinson power value to be $n=2$ ), for timber and glulam, the determining criterion becomes

$$
\frac{\sigma_{6}^{2}}{S^{2}}-\left(1-\frac{\sigma_{1}}{X}\right) \cdot\left(1+\frac{\sigma_{1}}{X^{\prime}}\right)-\left(1-\frac{\sigma_{2}}{Y}\right) \cdot\left(1+\frac{\sigma_{2}}{Y^{\prime}}\right)=-1,
$$

which is identical to Eq. 11 with $F_{12}=0$ and $1 / S^{2}=1 / X^{\prime} Y+$ $1 / Y^{\prime}$ :

$$
\frac{\sigma_{6}^{2}}{S^{2}}+\frac{\sigma_{1}}{X}-\frac{\sigma_{1}}{X^{\prime}}+\frac{\sigma_{1}^{2}}{X X^{\prime}}+\frac{\sigma_{2}}{Y}-\frac{\sigma_{2}}{Y^{\prime}}+\frac{\sigma_{2}^{2}}{Y Y^{\prime}}=1
$$

Because the virtual work equations, and thus the laws of limit analysis, do not apply for hardening, it also is necessary to use Eq. 33 in the codes in all cases for timber and 
clear wood to replace the now often used, but not valid Norris equations. ${ }^{9}$

\section{Discussion}

The failure criterion of wood consists of an orthotropic third-degree tensor-polynomial, Eq. 16, first given for wood with all aspects by van der Put. ${ }^{1}$ This polynomial is shown, for the same loading cases, to be identical to the $\mathrm{Wu}$ mixed-mode I-II equation, ${ }^{4}$ which is theoretically explained by van der $\mathrm{Put}^{3}$ in terms of hardening due to hindered microcrack extension. Thus, the theoretically explained equation, Eq. 16, can be stated to be the real failure criterion for wood.

The second-degree polynomial part of the failure criterion, Eq. 7 or Eq. 11, is shown to be the orthotropic critical distortional energy principle for initial yield showing the start of dissipation of distortional energy of the elastic stage, and thus is, by this minimal work principle, the exact initial yield criterion. After further loading, at final flow, $F_{12}=0$. This means an absence of coupling terms between the normal stresses. This is possible only when the reinforcement takes the whole normal loading, causing the matrix to fail by shear and the critical distortional energy principle thus reduces to the Tresca criterion. The necessary validity of the Tresca criterion is confirmed by van der Put ${ }^{10,11}$ where the strongly increased (sixfold) compression strength under the load of locally loaded blocks and the increased embedding strength of dowels is explained by the construction of the equivalent slip line field in the specimen using the Tresca criterion. In addition, the many apparent contradictions of the different investigations are explained by this theory. This strong increase of the compression strength is due to confined dilatation by real hardening (when the empty spaces in wood are pressed away). The Tresca criterion satisfies the normality rule and thus inherently the theorems of limit analysis for matrix failure. The normality rule thus need not apply for the orthotropic criterion. This condition is now shown to be replaced by the minimum work condition for dissipation represented by the yield equation and the hardening state constants $C_{d}$ and $C_{t}$ of Eq. 17 .

The distinction between the application of Eq. 10 or Eq. 11 simply follows from the exponent of the Hankinson equation (Eq. 34). Equation 14 shows that the exponent $n$ of this Hankinson power formula:

$$
\frac{\sigma_{t} \cos ^{n} \theta}{X}+\frac{\sigma_{t} \sin ^{n} \theta}{Y}=1
$$

is $n=2$ for tension and compression at yield when there are no higher-degree polynomial terms. A value of $n$, different from $n=2$ thus means the presence of higher-degree terms due to hardening after yield given by Eq. 16 .

The initial yield criterion of Eq. 13 or Eq. 14 should satisfy both the elastic and the yield conditions at the same time. Because the Hankinson power equation with $n=2$ also applies for the off-axis modules of elasticity and because this modulus is proportional to the strength, the Hankinson power equations with $n=2$, Eq. 14, satisfy this requirement. Thus, $n=2$ is necessary for initial yield. This does not only apply for clear wood, but it is mentioned also by Möhler ${ }^{12}$ that $n=2$ for lumber and for glued laminated wood. In Kollmann $^{6}$ (p. 686, 809), $n \approx 2$ is found for clear wood in bending and in tension and for the embedment strength on (p. 888). The combined shear-compression tests (of Keylwerth in Kollmann, ${ }^{6}$ p. 906) show, for off-axis longitudinal shear and in the radial plane, that $n=2$, showing no hardening terms for the shear strength. This also applies for the tangential plane according to the test method of Keenan and Jaeger, ${ }^{7}$ but in this case not for the radial plane. Early instability of uniaxial compression tests and strong hardening in the more stable biaxial compression tests follows from the data of Hemmer. ${ }^{2}$

In Eq. 17, the value of the hardening state parameter $C_{d}$ for compression or $C_{t}$ for tension, determines entirely the hardening state and by that the precise form of the measured Hankinson curve. This may also, as an empirical function of the stress level, determine the loading curves.

Because tests in longitudinal compression show other hardening rather than tests in tension and thus different terms, separate data fits for tension and compression are necessary. However, Eq. 32 for tension, also can be applied for compression, providing a better general equation than Eq. 33. Because of possible instability of structures at yield, hardening can be lacking (as in the oblique grain test) and the second-degree polynomial, or critical strain energy yield condition (Eq. 33), applies as the ultimate failure condition for the codes.

\section{Conclusions}

A derivation is given of the orthotropic extension of the critical distortional energy principle of yield, showing energy dissipation according to a minimum energy principle. This leads at final yield to an extended Tresca criterion for matrix failure. For stable tests, hardening is possible, leading to a general polynomial failure criterion of the fourth degree. This criterion can be resolved in two factors: for compression and for tension leading to extended Hankinson equations for uniaxial loading, making determination of all constants of the general failure criterion possible from simple uniaxial oblique-grain compression and tension tests instead of problematic biaxial tests. The extended Hankinson equations also show necessary relations between the constants of the general criterion, providing necessary datafitting conditions. For compression and for tension, the value of one hardening state constant fully describes the form of the hardening curve at different hardening stages, providing the possibility of a new hardening description. The normality rule does not apply for orthotropic wood because the matrix is determining initially. Because dissipation at yield is according to a minimum energy principle, this rule can be replaced by the hypothesis of intrinsic minimum work. 


\section{References}

1. van der Put TACM (1982) A general failure criterion for wood Proceedings of 15th Conseil Industrielle des Bois-International Union of Forestry Research Organizations Meeting, Boras, Sweden

2. Hemmer K (1985) Versagensarten des Holzes der Weisstanne unter mehrassige Beanspruchung. Dissertation, University of Karlsruhe, Karlsruhe

3. van der Put TACM (2007) A new fracture mechanics theory of orthotropic materials like wood. Eng Fract Mech 74:771-781

4. Wu EM (1967) Application of fracture mechanics to anisotropic plates. J Appl Mech 34:967-974

5. Gopu VKA (1987) Validity of distortion-energy-based strength criterion for timber members. J Struct Eng 113:2475-2487

6. Kollmann F (1951) Technologie des Holzes und der Holzwerkstoffe. Springer, Berlin Heidelberg New York, p 686, 809, 905
7. Keenan FJ, Jaeger TA (1978) Effect of transverse stress on shear strength and failure mechanism of Douglas fir. Proceedings of the 1st International Conference on Wood Fracture, Banff, Alberta, Canada

8. Tsai SW, Wu EM (1971) A general theory of strength for anisotropic materials. J Comp Mater 5:58-80

9. Norris CB (1939) The elastic theory of wood failure. Trans ASME 61:259-261

10. van der Put TACM (2008) Derivation of the bearing strength perpendicular to the grain of locally loaded timber blocks. Holz Roh Werkst 66:409-417

11. van der Put TACM (2008) Explanation of the embedding strength of particle board. Holz Roh Werkst 66:259-265

12. Möhler K (1978-1979) Consideration of combined stresses for lumber and glued laminated timber CIB-W18-9-6-4, 1978 en CIBW18-11-6-3, 1979 